Research Paper

\title{
Experiences of Single Incision Cholecystectomy
}

\author{
Huseyin Yilmaz ${ }^{1}$, Husnu Alptekin ${ }^{1}$, Fahrettin Acar ${ }^{1}$, Ilhan Ciftci ${ }^{\circledR}$, Ahmet Tekin ${ }^{3}$, Mustafa Sahin ${ }^{1}$ \\ 1. Department of General Surgery, Selcuklu Medical School, Selcuk University, TURKEY; \\ 2. Department of Pediatric Surgery, Selcuklu Medical School, Selcuk University, TURKEY; \\ 3. Department of General Surgery, Meram Medical School, Necmettin Erbakan University, TURKEY.
}

$\square$ Corresponding author: Ilhan Ciftci, M.D. Selcuk Universitesi Selcuklu Tip Fakultesi, Cocuk Cerrahisi A.D. Konya, Turkey. E-mail: driciftci@yahoo.com Tel: 00903322415000 Fax: 00903322412184.

(C) Ivyspring International Publisher. This is an open-access article distributed under the terms of the Creative Commons License (http://creativecommons.org/ licenses/by-nc-nd/3.0/). Reproduction is permitted for personal, noncommercial use, provided that the article is in whole, unmodified, and properly cited.

Received: 2012.08.13; Accepted: 2012.11.26; Published: 2012.12.19

\begin{abstract}
Purpose: Single incision laparoscopic surgery in suitable cases is preferred today because it results in less postoperative pain, a more rapid recovery period, more comfort, and a better cosmetic appearance from smaller incisions. This study aims to present our experiences with single incision laparoscopic cholecystectomy to evaluate the safety and feasibility of this procedure.

Methods: A total of 150 patients who underwent single incision laparoscopic cholecystectomy between January 2009 and December 20I I were evaluated retrospectively. In this serial, two different access techniques were used for single incision laparoscopy.

Results: Single incision laparoscopic cholecystectomy was performed successfully on 150 patients. Median operative time was 29 (minimum-maximum=5-66) minutes. Median duration of hospital stay was found to be 1.33 (minimum-maximum=I-8) days. Patients were controlled on the seventh postoperative day. Bilier complication was not seen in the early period. Five patients showed port site hernia complications. Other major complications were not seen in the 36-month follow-up period.

Conclusion: Operation time of single incision laparoscopic cholecystectomy is significantly shortened with the learning curve. Single incision laparoscopic cholecystectomy seems a safe method.
\end{abstract}

Key words: Cholecystectomy, Single incision

\section{Introduction}

With the development of minimally invasive surgical techniques, surgeons are now focused on achieving cosmetic results and significantly reducing surgical trauma (1). Today, laparoscopic surgery is preferred because it involves less postoperative pain, a rapid recovery period, a greater degree of comfort, and a better cosmetic appearance from smaller incisions. Laparoscopic cholecystectomies in particular require shorter operation times and involve fewer complications as surgeons' experience with them increases (2). Novel methods are currently being attempted to further develop the well-known advantages of this procedure so that the incision size and the number of trochars can be reduced (3).

Although developing laparoscopic cholecystectomy is very difficult today, many surgical procedures are currently done with a single port or incision. Many recent publications have reported that cholecystectomies traditionally performed through four ports are now being done successfully with fewer ports. The single incision laparoscopic cholecystectomy (SILC) was first described in 1997 as involving two incisions done in the periumbilical region (4). Specific hand tools and ports are not needed in many single incision laparoscopic cholecystectomies. The literature provides various definitions such as SILS 
(single incision laparoscopic surgery), single-port access, single laparoscopic incision transabdominal surgery, dual incision laparoscopic technique, and single incision multiport laparoscopic cholecystectomy (5-6).

The advantages of SILC include less postoperative pain, faster return to daily activities, and a better cosmetic outcome. Patients' demand for better cosmetic outcomes and minimally invasive surgeries plays a significant role in increasing the popularity of this technique. A better cosmetic result makes it possible for the patient might prefer this operation. (7). Published series are not available for preoperative safe dissection and manipulation difficulty, higher cost, and postoperative complications such as hernia could emerge depending on the size of the incision (8).

This study aimed to present 150 cases of SILC performed between January 2009 and December 2011 to evaluate the feasibility of the procedure.

\section{Patients and Method}

\section{Patients}

One hundred fifty patients (47 men, 103 women) who were selected to undergo SILC between January 2009 and December 2011 were evaluated retrospectively. After receiving institutional review board approval (no: 2012/21), follow-up data were obtained from hospital charts and office records. The patients ranged in age from 14 to 87 years old (mean age, 44 years). All patients consented to the laparoscopic cholecystectomy. They were informed about the operative strategy of having a single incision in the abdomen with the possibility of requiring several more incisions or conversion to an open technique if indicated. Inclusion criteria were the presentation with typical symptoms of biliary colic and cholelithiasis, as indicated on ultrasonography. No patients declined the technique. Patients underwent SILC for several conditions: gallstones ( $\mathrm{n}=137$ cases), acute cholecystitis from gallbladder stones ( $\mathrm{n}=10$ cases), and cholesterol polyps ( $\mathrm{n}=3$ cases). The median body mass index (BMI) was 29.1 (minimum-maximum=22-33) $\mathrm{kg} / \mathrm{m}^{2}$. All cases were confirmed by ultrasonography. The exclusion criteria were as follows: American Society of Anesthesiologists (ASA) classification IV, the need for an additional port, conversion to open surgery, and suspicion of malignancy.

Of the 150 patients, 23 had a history of abdominal operations. Among the 23 operations, 12 were cesarean sections, four were appendectomies, six were total hysterectomies, and one was an umbilical hernia.
Oral food intake was ceased eight hours before the operation; antibiotic prophylaxis was done peroperatively with a first-generation cephalosporin.

All patients were evaluated for operative time. Gender, age, body mass index (BMI), ASA classification, abdominal operation history, pathological results, intraoperative description, hospital stay, intraoperative washing, intraoperative complications, and postoperative complications were included in the present study for analysis.

\section{Operative Procedures}

Two different access techniques were used for single incision laparoscopy. Each patient was placed in a supine position, with the head raised to 30 degrees and the right side up. The surgeon stood between the patient's legs. A camera assistant stood next to the patient's left leg. In all cases, skin and subcutaneous tissues were passed with a $20 \mathrm{~mm}$ transverse incision in a standard manner.

In the first 30 cases, the abdomen was exposed without using a veres needle to provide a healthy fascia between trochars using two $5 \mathrm{~mm}$ and one 10 $\mathrm{mm}$ trochar with a multifascial puncture technique (9-10). Three ports were placed in the abdominal cavity (Figure 1A). An integrated rigid 30-degree, $5 \mathrm{~mm}$ laparoscope, and 5/10 mm laparoscopic instruments were selected for all procedures. Then, the abdomen was insufflated to a continuous $13 \mathrm{mmHg}$. The gallbladder was slinged from the fundus using an intra-abdominal suture with a straight needle $2 / 0$ prolene entering through the right subcostal margin and fixed to the abdominal wall. To provide a "critical view," a Hartman pouch was slinged with a second intra-abdominal suture entering through the right upper quadrant of the abdomen. These two intra-abdominal sling sutures took the place of graspers used in a standard cholecystectomy and provided better visualization for gallbladder manipulation and Calot's triangle. The cystic artery and the cystic duct were clipped with a $5 \mathrm{~mm}$ clip and were divided with endoscopic scissors. The gallbladder was separated with the aid of a hook cautery and removed after trochar defects of the fascia were binded. An endobag was not used in any of the patients to remove the gallbladder, and the gallbladder was removed through an umbilical incision. Fascial defects were closed using interrupted $2 / 0$ silk sutures. The skin was sutured in an intradermic fashion with rapid Vicryl.

As the second access technique, skin and subcutaneous tissues were passed through with a $20 \mathrm{~mm}$ transverse incision from the umbilicus in 120 subsequent patients. Fascia was slinged with notched 
clamps, and the abdomen was entered using a transverse fascia incision. A port manufactured for SILS (SILS port Covidien $($ ) ) was placed (Figure 1B). Intra-abdominal pressure was elevated to $13 \mathrm{mmHg}$ with insuflation. The abdomen was entered above the specific port with two $(5 \mathrm{~mm})$ and one $(12 \mathrm{~mm})$ trochars. A $5 \mathrm{~mm}, 30^{\circ}$ laparoscope was used. The gallbladder was suspended using the grasper. The cystic artery was dissected, taking special care with the dissection of the cystic duct. The relationship between the main bile duct and the cystic duct was displayed. The artery and the cystic duct were clipped with a medium clip from a $12 \mathrm{~mm}$ trochar (Figure 1D) and divided with endoscopic scissors. The gallbladder was separated from the liver using a hook cautery and removed from the incision line. An endobag was not used in any of the patients. A laparoscopic exploration was then performed to ensure that no intraoperative complications occurred, such as bleeding or biliary leakage. After removing the port and releasing the residual carbon dioxide, the fascia defect was closed with loop prolene. The skin was sutured in an intradermic fashion using rapid Vicryl.

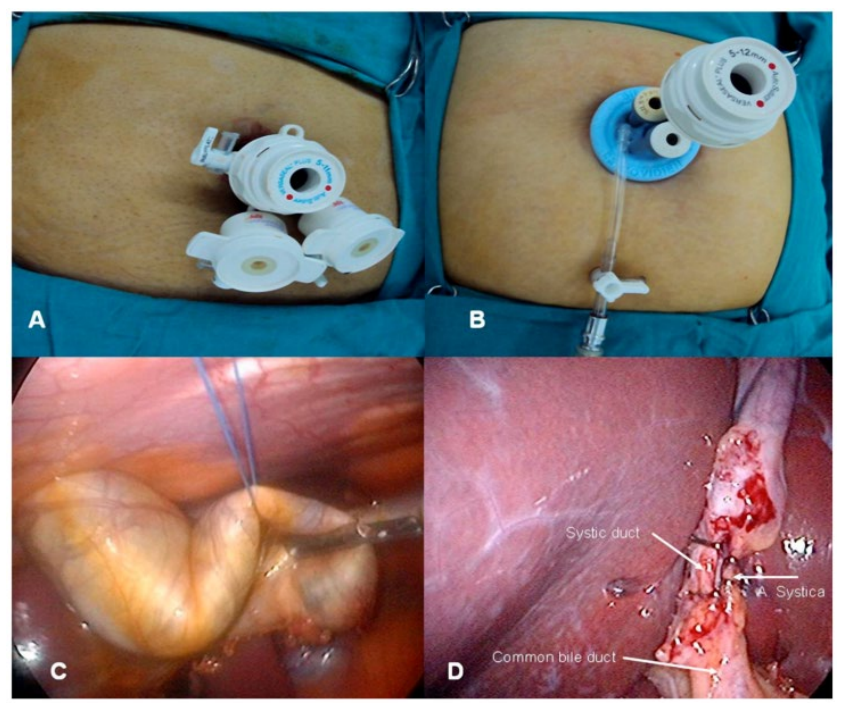

Figure I. Three ports were placed in the abdominal cavity (A), port manufactured for SILS was placed (B), suture was leads to bile leak (C), artery and cystic duct were clipped (D).

\section{Statistical Analysis}

Statistical analysis was performed using the Statistical Package for the Social Sciences (SPSS Inc., Chicago, IL) version 18 for Windows (Microsoft Corp., Redmond, WA). Statistical significance was set at $\mathrm{p}<0.05$. The Kruskal-Wallis test was used as a descriptive statistical method to examine the qualita- tive data, and the chi-square test was used for the quantitative data analysis.

\section{Results}

A total of 158 patients underwent SILC between January 2009 and December 2011. Patient characteristics are shown in Table 1 . The procedure was performed successfully on 150 patients. For the remaining eight patients, the operation was completed by entering an additional port. An additional port was required for two patients due to bleeding from the cystic artery, for two other patients due to difficulties with dissection attributable to acute cholecystitis, and for the last four patients due to an inability to provide adequate intra-abdominal pressure as a result of being unable to perform the multifascial puncture technique. These eight patients were thus unable to successfully complete SILC.

Table I. Characteristic of I50 Patients Undergoing Single Incision Laparoscopic Surgery for Cholecytectomy.

\begin{tabular}{lll}
\hline & Total $(\mathrm{n}=150)$ & $\begin{array}{l}\text { First 30 cases } \\
(\mathrm{n}=30)\end{array}$ \\
\hline Age & $44 \pm 7.8$ & $41 \pm 7,6$ \\
Male/Female & $47 / 103$ & $5 / 25$ \\
Operative technique & $\begin{array}{l}\text { Multipuncture and } \\
\text { SILS port }\end{array}$ & $\begin{array}{l}\text { Multipuncture } \\
\text { and SILS port } \\
\text { Operative time minute (medi- }\end{array}$ \\
$29(5 / 66)$ & $49(39 / 66)$ \\
an, min/max) & 23 & \\
Previous abdominal surgery & 23 & - \\
Appendectomy & 4 & 2 \\
Cesarean section & 12 & - \\
Total hysterectomy & 6 & - \\
Umbilical hernia & 1 & 30 \\
Diagnosis & & - \\
Gallstones & 137 & - \\
Acute cholecystitis & 10 & 1.52 \\
Cholesterol polyps & 3 & \\
Hospital stay & 1.33 &
\end{tabular}

The median operative time, which was measured as the time required to insert the trocar and close the wound, was 33 minutes (minumum-maximum=10-71). Postoperative intra-abdominal organ injuries, bleeding, and biliary system damage were not observed in any of the patients. To define the learning curve for surgeons experienced in similar cases were consecutively.

All patients were discharged within a postoperative median of 1.33 days (minumum-maximum=1-8) and invited for control on postoperative day seven. Complications that did not 
develop during the early period, such as hematoma, herniation, subcutaneous emphysema, and deep vein thrombosis, were found. Five patients treated with oral antibiotics had superficial wound infections. Port-site hernias (PSH) developed within two months in two patients and within six months in three other patients. Two patients were normal weight experienced PSH. Of these patients, a 61 year-old woman had chronic obstructive pulmonary disease; and a 43 year-old man had wound infection. One patient was the overweight experienced PSH.this patient a 43 year-old women who had wound infection. The diagnosis of a hernia was confirmed by ultrasound and patients underwent elective hernia repair with mesh implantation. During 36 months of postoperative follow-up, no bile duct injuries, bleeding, or bile fistulas were seen. The incisions healed well in the all patient.

\section{Discussion}

Multiport cholecystectomies, which were traditionally performed through four ports, are now being done successfully with fewer ports. Many techniques have been described to reduce the port number. In 1997, SILC was first described as involving two incisions done in the periumbilical region (4). Although the procedure has had many names, SILC is the most widely accepted name. SILS on a more limited area of the stomach, colon, or other natural orifices can be done using special equipment by entering into the Natural Orifice Translumenal Endoscopic Surgery (NOTES) from the more commonly accepted. NOTES gastrotomy may occur after potential complications from leakage, and specific tools, cost because SILS has overshadowed the difficulty of learning. The most important advantage of SILC is that it can be completed using conventional laparoscopic instruments. SILC began to increase in popularity following the introduction of newly developed special ports, camera systems providing better visualization, and tools with increased motion ability. For better monitoring of the learning phase of Calot's triangle laparoscopic flexible use, the gallbladder taken by the suspension of prolene abdominal wall slinging, although listed as an additional cost of angled dissector to use as tool to increase the experience gained with conventional laparoscopic equipment is adequate. Given the increase in the experience of our clinic, SILS can be successfully performed for many intra-abdominal surgeries (e.g., cholecystectomy, appendectomy, splenectomy, nissen funduplication, obesity surgery, and adrenalectomy), as reported in the current literature (11).

Acute cholecystitis is the other problem in laparoscopic cholecystectomy (LC). Ten patients had a diagnosis of acute cholecystitis, and the time that elapsed between onset of the symptoms and operation was less than 48 hours. SILC was completed without complications in all 10 of these patients in spite of the difficulties involved in dissecting Calot's triangle.

Preoperative ultrasonography was performed routinely on all patients in our study. The specificity and sensitivity of ultrasonography for benign gallbladder diseases are very high. An increase in the thickness of the gallbladder wall, gallbladder neoplasms, and the main biliary ducts give sufficient information about the status of the ultrasonography. Pericholecystic adhesions related to previous cholecystitis episodes and preoperative acute cholecystitis in particular are the main factors that determine operative time in patients planning to undergo SILC.

In terms of the key findings of our study, no significant difference was seen between groups in terms of their length of hospital stay; median hospital stay duration was 1.33 days. SILC may also be performed as an outpatient surgery, and such patients should be monitored closely for peritoneal signs (12).

One important finding in our study was that the operative time shortened significantly with the surgeons' learning curve as they gained more experience with SILC. Median operative time was 49 minutes (minimum-maximum $=39-66$ ) in the first 30 cases and 34 minutes (minimum-maximum=15-59) in the next 30 cases. This difference was statistically significant $(\mathrm{p}$ $<0.01$ ). In our series, the first 30 cases were considered part of the training phase. The reason for the longer average operation time in the first group was the difficulty that surgeons with multi-puncture laparoscopic cholecystectomy (MPLC) experience face during SILC. During this initial phase, SILC dissector and grasping forceps were used to enter a single incision and were placed parallel to each other; insufficient experience in the use of a cross-dissector and grasping forceps led to dissection difficulty. MPLC manipulation was difficult because of the Hartman pouch, and the laparoscope and surgical instruments overlapped with each other. In addition, we experienced an air leak using the multipuncture technique, and intra-abdominal pressure did not increase. Operating time significantly shortened as the surgeons' SILC experience increased. In recent articles, Duron et al. reported a series of 55 cases performed in a single institution in which a "learning curve" effect was present with regard to shorter operating times and the inclusion of more technically difficult patients as the surgeons gained experience with the procedure (13). Mutter et al. analyzed the implementation of this type of surgery in a teaching hospital by comparing six 
surgeons (three senior surgeons and three junior surgeons). They found no significant difference between operating times and complication rates, thus advocating for the safe implementation of SILC in teaching hospitals (14). Another study we conducted previously shown that single-port cholecystectomies and traditional laparoscopic cholecystectomies did not have any statistically significant differences in terms of operating time, length of hospital stay, and conversion to open surgery (8).

Gangl et al. gathered SILC patient data prospectively and compared them with matched controls from a group of 163 patients who underwent MPLC during the same period, with no significant differences in age, gender, BMI, ASA classification, diagnosis of acute cholecystitis, or previous abdominal surgeries (15). They reported a SILC cholecystectomy completion rate of $85.1 \%$, with conversion to MPLC in nine patients and an open cholecystectomy in one patient due to inadequate visualization of the anatomy, versus a $100 \%$ completion rate in the LC group with no significant differences with regard to postoperative pain, analgesic use, length of stay, or complications. The only significant difference was the length of surgery, with a longer operating time in the SILC cholecystectomy group (75 min versus $63 \mathrm{~min}$ ). They concluded that SILC is comparable to MPLC, although SILC is associated with a longer operating time (15-16).

We used a port manufactured specifically for single incision laparoscopic surgery in 120 cases and observed that tool manipulation was easier than it is for multifascial puncture, and there was less superposition of tools. The most important factor for superposition of the camera and tools used in a multifascial puncture is big trochar heads. Using head-free trochars in this technique significantly eliminates this problem. According to our experience, camera-hand tool superposition and manipulation difficulties are minimized by correctly positioning the surgeon and the camera assistant. In our study, the surgeon stood between the patient's legs, and the camera assistant stood next to the patient's left.

The cystic artery and the cystic duct should be exposed clearly and removed safely to prevent complications such as bleeding and bile duct injury. Slinging the Hartman pouche and fundus with sutures facilitate dissection and provide clear exposure of the cystic artery and the cystic duct for safe dissection. However, this always causes bile to leak from the gallbladder, which can decrease the safety of the procedure (Figure 1C). Bile leakage from the sutures must be washed to prevent postoperative peritoneal irritation. Slinging the gallbladder with a suture pro- longs the operating time and causes unnecessary abdominal irrigation. Another technique is retracting the Hartman with an articulating grasper without suturing and dissecting Calot's triangle. After the first 30 cases, we completed operations without slinging the gallbladder with sutures. We observed that this method shortened the operating time and made dissection easy. Thus, use of the intra-abdominal sutures is not mandatory and depends on the preference of the surgeon.

One of the late complications of SILC, incisional hernias, was detected in five patients during the median 23-month (minimum-maximum=1-36) follow-up period. All of the hernias occurred in the first six months after the operation. Helgstrand et al. reported that the rate of trocar hernia development was $1.6 \%$ for laparoscopic cholecystectomies in their series (17). These results indicate that the risk of incisional hernia development is high in SILC. In an original report of Marks et al., no significant difference existed with respect to complications (18). However, in the largest case series published to date by Phillips et al., the number of complications associated with single incision surgery increased as the number of patients increased (19). In theory, the learning curve has leveled off, indicating that complications are inherent in the procedure, making the feasibility of widespread SILC application questionable. One of the main complications is the increased risk of postincisional hernias after SILS due to the increase in the size of the fascia defects. An attempt has been made to avoid this by turning multiple fascial defects into a single incision; however, the results were inconclusive $(8,16,18$, $19,20)$.

\section{Conclusion}

Based on the present findings, SILC seems to may be a safe method. The operating time is long initially, but it shortens as surgeons become more experienced with the procedure. However, patients should be informed about the risks of port-site hernia and instructed to avoid heavy exercise during the first six months post-operation.

\section{Competing interests}

The authors have declared that no competing interest exists.

\section{References}

1. Luo C, Yang Q, Liu B, et al. Difficulties and countermeasures of transumbilical single incision laparoscopic cholecystectomy. J Am Coll Surg. 2012 May; 214(5):e35-8.

2. Durkin ET, Shaaban AF. Recent advances and controversies in pediatric laparoscopic surgery. Surg Clin North Am. 2008 Oct; 88(5):1101-19. 
3. Alptekin H, Sahin M. Gallbladder bed irrigation with bupivacaine improves pulmonary functions after laparoscopic cholecystectomy. Langenbecks Arch Surg 2010; 395:501-504.

4. Navarra G, Pozza E, Occhionorelli S, et al. One-wound laparoscopic cholecystectomy. Br J Surg 1997; 84(5):695.

5. Cuesta MA, Berends F, Veenhof AA. The "invisible cholecystectomy": A transumbilical laparoscopic operation without a scar. Surg Endosc. 2008 May; 22(5):1211-3.

6. Merchant AM, Cook MW, White BC, et al. Transumbilical Gelport access technique for performing single incision laparoscopic surgery (SILS). J Gastrointest Surg. 2009 Jan; 13(1):159-62.

7. Garg P, Thakur JD, Singh I, et al. A Prospective Controlled Trial Comparing Single-incision and Conventional Laparoscopic Cholecystectomy: Caution Before Damage Control. Surg Laparosc Endosc Percutan Tech. 2012 Jun; 22(3):220-5.

8. Alptekin H, Yilmaz H, Acar F, et al. Incisional hernia rate may increase after single-port cholecystectomy. J Laparoendosc Adv Surg Tech A. 2012 Oct; 22(8):731-7.

9. Edwards C, Bradshaw A, Ahearne P, et al. Single-incision laparoscopic cholecystectomy is feasible: initial experience with 80 cases. Surg Endosc 2010; 24(9):2241-2247.

10. Piskun G, Rajpal S. Transumbilical laparoscopic cholecystectomy utilizes no incisions outside the umbilicus. J Laparoendosc Adv Surg Tech A. 1999 Aug; 9(4):361-4.

11. Barbaros U, Sümer A, Tunca F, et al. Our early experiences with single-incision laparoscopic surgery: the first 32 patients. Surg Laparosc Endosc Percutan Tech. 2010 Oct; 20(5):306-11.

12. Erbella J Jr, Bunch GM. Single-incision laparoscopic cholecystectomy: the first 100 outpatients. Surg Endosc. 2010 Aug; 24(8):1958-61.

13. Duron VP, Nicastri GR, Gill PS. Novel technique for a single-incision laparoscopic surgery (SILS) approach to cholecystectomy: single-institution case series. Surg Endosc. 2011 May; 25(5):1666-71.

14. Mutter D, Callari C, Diana M, et al. Single port laparoscopic cholecystectomy: which technique, which surgeon, for which patient? A study of the implementation in a teaching hospital. J Hepatobiliary Pancreat Sci. 2011 May; 18(3):453-7.

15. Gangl O, Hofer W, Tomaselli F, et al. Single incision laparoscopic cholecystectomy (SILC) versus laparoscopic cholecystectomy (LC)-a matched pair analysis. Langenbecks Arch Surg. 2011 Aug; 396(6):819-24.

16. Arroyo JP, Martín-Del-Campo LA, Torres-Villalobos G. Single-incision laparoscopic cholecystectomy: is it a plausible alternative to the traditional four-port laparoscopic approach? Minim Invasive Surg. 2012; 2012:347607.

17. Helgstrand F, Rosenberg J, Kehlet H, et al. Low risk of trocar site hernia repair 12 years after primary laparoscopic surgery. Surg Endosc 2011; 25(11):3678-3682.

18. Marks J, Tacchino R, Roberts K, et al. Prospective randomized controlled trial of traditional laparoscopic cholecystectomy versus single-incision laparoscopic cholecystectomy: report of preliminary data. Am J Surg. 2011 Mar; 201(3):369-72.

19. Phillips MS, Marks JM, Roberts K, et al. Intermediate results of a prospective randomized controlled trial of traditional four-port laparoscopic cholecystectomy versus single-incision laparoscopic cholecystectomy. Surg Endosc. 2012 May; 26(5):1296-303.

20. Antoniou SA, Pointner R, Granderath FA. Single-incision laparoscopic cholecystectomy: a systematic review. Surg Endosc. 2011 Feb; 25(2):367-77. 